\title{
Vigor de sementes de girassol pela análise computadorizada de plântulas
}

\section{Sunflower seed vigor determined by computerized seedling analysis}

\author{
Carla Massimo CALDEIRA ${ }^{1,2}$; Maria Laene Moreira de CARVALHO ${ }^{3}$; João Almir OLIVEIRA ${ }^{4}$; \\ Stefânia Vilas Boas COELHO ${ }^{5}$; Verônica Yumi KATAOKA \\ ${ }^{1}$ Parte da dissertação de mestrado do primeiro autor; Pesquisa financiada pelo Conselho Nacional de \\ Desenvolvimento Científico e Tecnológico, CNPq \\ ${ }^{2}$ Autor para correspondência. Doutora, Federal de Lavras, UFLA, Departamento de Agricultura, Universidade, \\ Caixa Postal 3740, 37200-000 - Lavras, MG, Brasil, carlaufla@yahoo.com.br \\ 3 Doutora, Universidade Federal de Lavras, UFLA, mlaenemc@dag.ufla.br \\ 4 Doutor, Universidade Federal de Lavras, UFLA, jalmir@dag.ufla.br \\ 5 Eng. Agrônoma, Universidade Federal de Lavras, UFLA, stecoelho456@hotmail.com \\ ${ }^{6}$ Doutora, Universidade Estadual de Santa Cruz, UESC, veronicayumi@terra.com.br
}

Recebido em: 24-01-2014; Aceito em: 14-06-2014

\begin{abstract}
Resumo
O Seed Vigor Imaging System (SVIS®) tem-se destacado, tanto pela rapidez quanto pela eficiência para a avaliação do potencial fisiológico de sementes de várias espécies. O teste estima o vigor de sementes por meio de parâmetros relacionados ao crescimento e à uniformidade das plântulas, calculados a partir do processamento de imagens digitais obtidas no teste de germinação. O objetivo deste trabalho foi verificar a eficiência do sistema computadorizado para análise de imagens de plântulas SVIS $\circledast$, desenvolvido para soja, para a determinação do vigor de sementes de girassol. Seis lotes de sementes recém-colhidas de girassol (cultivar Helio 251) foram submetidos aos testes de germinação, emergência, envelhecimento acelerado, tetrazólio e sanidade. O SVIS® forneceu dados referentes ao índice de vigor, de uniformidade de desenvolvimento, de crescimento e ao comprimento de plântulas, para plântulas escaneadas com 2 e 3 dias de germinação. Os seis lotes foram avaliados após a colheita, e depois de quatro meses, armazenados em condição de armazém convencional $\left(25 \pm 2^{\circ} \mathrm{C}\right)$. A análise computadorizada de imagens de plântulas provenientes do teste de germinação com o uso do software SVIS® não apresenta resultados compatíveis com os demais testes de avaliação da qualidade das sementes.
\end{abstract}

Palavras-chave adicionais: análise de imagem; Helianthus annus L; qualidade fisiológica; teste de vigor.

\begin{abstract}
The Seed Vigor Imaging System (SVIS ${ }^{\mathrm{TM}}$ ) has stood out for being both fast and efficient to evaluate the physiological potential of seeds of various species. The test evaluates the vigor of seeds by means of parameters related to seedlings growth and uniformity which are calculated from the processing of digital images of the germination test. The objective of this study was to verify the accuracy of the computerized system for seedling image evaluation for the determination of sunflower seed vigor. Six recently harvested sunflower seed lots (cultivar Helio 251) were submitted to the germination, emergence, accelerated aging, tetrazolium, and sanitary tests. The (SVIS ${ }^{\mathrm{TM}}$ ) supplied reference data concerning vigor index, seedling uniformity, growth, and length for seedlings scanned 2 and 3 days after the start of the test. The six lot seeds were evaluated at harvest and after four months of storage under conventional conditions $\left(25 \pm 2^{\circ} \mathrm{C}\right)$. The results of computerized analysis of images of seedling resulting from a germination test are not compatible with the results of the tests used in this experiment to evaluate sunflower seed physiological quality.
\end{abstract}

Additional keywords: Helianthus annus L; image analysis; physiological quality; vigor test.

\section{Introdução}

O girassol (Helianthus annuus L.) tem importância para a economia mundial e juntamente com a soja e a canola são as três mais importantes culturas anuais produtoras de óleo do mundo (FAGUNDES, 2009). No Brasil, o cul- tivo do girassol tem aumentado significativamente nos últimos anos, principalmente em cultivos de safrinha, visando a atender à produção de biodiesel e ao mercado de óleo comestível. Entretanto, para que a ampliação da oferta dessa matéria-prima seja bem-sucedida, é necessário uso de tecnologias, como a produção de 
sementes de qualidade, imprescindíveis para a manutenção da produtividade. Dentro desse contexto, a adequação de técnicas que sejam rápidas, práticas e eficientes para avaliação da qualidade de sementes e que facilitem a interpretação dos resultados e o controle de qualidade em diversos segmentos da produção de sementes é importante (MARCOS FILHO et al., 2009; VIEIRA et al., 1999).

SAKO et al. (2001) desenvolveram um programa destinado à determinação do vigor de sementes de alface, mediante a análise computadorizada de imagens de plântulas (Seed Vigor Imaging System - SVIS®). O sistema permite efetuar a análise relativamente rápida de plântulas, mediante seu processamento e cálculos de um índice de vigor e de uniformidade de desenvolvimento, ambos expressos numericamente (valores de 0 a 1.000), e avaliação do comprimento de plântulas ou parte delas. O sistema automatizado possui amplo potencial para a determinação do vigor com diferentes alternativas de aplicação e obtenção de informações consistentes. A vantagem deste sistema inclui a rápida obtenção de resultados e a precisão, uma vez que não envolvem erros humanos de avaliação de cálculo, estimativas e medições (HOFFMASTER et al., 2003, 2005; SAKO et al., 2001).

A avaliação da qualidade de sementes por meio dos índices obtidos pelo sistema de análise de imagens tem sido empregado com sucesso para várias espécies, como soja (HOFFMASTER et al., 2003), alface (PEÑALOZA et al., 2005) e melão (MARCOS FILHO et al., 2006). Entretanto, a eficiência da utilização do teste para a investigação do vigor depende da adequação do sistema para a cultura do girassol, uma vez que não existe software específico para a espécie em questão. Assim, objetivou-se com esta pesquisa verificar a eficiência do sistema computadorizado de avaliação do vigor de plântulas (SVIS®) na avaliação do vigor de sementes de girassol.

\section{Material e métodos}

A pesquisa foi conduzida no Laboratório de Análise de Sementes do Departamento de Agricultura, da Universidade Federal de Lavras (UFLA), e no Laboratório de Análise de Imagens do Departamento de Produção Vegetal, da Escola Superior de Agricultura "Luiz de Queiroz".

Foram utilizados seis lotes de sementes recém-colhidas de girassol (cultivar Helio 251) submetidos a testes de vigor tradicionais e ao teste de germinação. Os resultados obtidos foram comparados com os calculados pelo sistema computadorizado SVIS\& (Seed Vigour Imaging System). Os seis lotes foram avaliados após a colheita e depois de quatro meses de armazenamento, em condição de armazém convencional $\left(25 \pm 2{ }^{\circ} \mathrm{C}\right)$.
Para a captura e o processamento das imagens, foi realizado o teste de germinação com quatro repetições de 25 sementes colocadas para germinar em duas fileiras paralelas sobre a superfície do papel toalha tipo "Germitest", na forma de rolo. O papel foi previamente umedecido com água destilada na proporção de 2,5 vezes sua massa. As sementes foram mantidas em câmara de germinação tipo BOD regulada a 25 C. Foram realizadas duas avaliações, com plântulas escaneadas com 2 e 3 dias de germinação. As imagens foram captadas em escâner HP Scanjet 2004 instalado de maneira invertida no interior de uma caixa de alumínio com $60 \mathrm{~cm}$ x $50 \mathrm{~cm}$ x $12 \mathrm{~cm}$ e operado por software Photosmart, com resolução de 98 dpi.

As plântulas de cada repetição foram transferidas do papel para uma folha de cartolina de cor preta com $30 \mathrm{~cm} \times 22 \mathrm{~cm}$ (correspondente ao tamanho da área útil do escâner), colocada sobre a plataforma interna da caixa metálica. As plântulas foram distribuídas de forma aleatória, sem distribuição organizada, tomando cuidado para que uma não interpusesse à outra. As imagens foram digitalizadas e analisadas pelo software Seed Vigor Imaging System (SVIS®), desenvolvido para a cultura da soja (HOFFMASTER et al., 2003). Durante a análise, - eixo raiz-hipocótilo de cada plântula foi marcado em vermelho pelo sistema e, para a maioria das plântulas, o eixo foi identificado corretamente. Houve, no entanto, necessidade de se efetuarem correções manuais de erros de digitalização (com auxílio do "mouse"), como a complementação de parte de plântulas marcadas parcialmente, exclusão de plântulas com anormalidades extremas e inclusão de plântulas não computadas. Após a análise e a avaliação de cada plântula, o software gera automaticamente valores numéricos referentes a um índice de vigor (valores de 0 a 1.000, diretamente proporcionais ao vigor), de uniformidade de desenvolvimento (0 a 1.000) e de crescimento (também de 0 a 1.000), além do comprimento de plântulas (através do posicionamento do mouse sobre cada plântula, indicando automaticamente o valor correspondente ao comprimento da plântula demarcada, em milímetros).

Estipulou-se para o tamanho máximo das plântulas, com base na média dos maiores comprimentos, os valores de $3,75 \mathrm{~cm}$ para as plântulas com 2 dias de germinação e $8,75 \mathrm{~cm}$ para 3 dias de germinação (plântulas com comprimento de $0 \mathrm{~cm}$ geraram índice de crescimento 0 , e plântulas com $3,75 \mathrm{~cm}$ aos 2 dias e $8,75 \mathrm{~cm}$ aos 3 dias geraram o índice de crescimento 1.000). A combinação testada para a obtenção do índice de vigor foi $70 \%$ crescimento (C) e 30\% uniformidade (U). Os valores do índice de vigor são baseados na velocidade e uniformidade de 
desenvolvimento das plântulas de acordo com o possível crescimento (valor) máximo, estipulado para a cultura (HOFFMASTER et al., 2003).

Para a determinação da qualidade dos lotes e comparação com os resultados da análise computadorizada, foram realizados os testes de emergência de plântulas, germinação, envelhecimento acelerado, sanidade e tetrazólio.

$O$ teste de emergência foi realizado utilizando 200 sementes por lote, divididas em quatro repetições. As 50 sementes de cada repetição foram colocadas em bandejas de plástico com dimensões de $60 \mathrm{~cm} \times 40 \mathrm{~cm} \times 10 \mathrm{~cm}$, contendo como substrato areia + terra, na proporção de $2: 1$, e retenção de água de $70 \%$. As bandejas com as sementes foram mantidas em câmara de crescimento regulada para $25^{\circ} \mathrm{C}$. As avaliações de plântulas emersas foram feitas aos 7 dias, para a obtenção da Primeira Contagem de Emergência, e 14 dias após a montagem do teste, para a Emergência Final. $O$ Índice de Velocidade de Emergência (IVE) foi calculado com os dados obtidos das contagens diárias do número de plântulas emersas, segundo MAGUIRE (1962).

Para o teste de germinação, foram utilizadas oito repetições de 25 sementes por lote. $\mathrm{O}$ substrato empregado foi rolo de papel do tipo "Germitest", umedecido com água destilada na proporção de 2,5 vezes a massa do substrato. As sementes foram postas para germinar em câmara de germinação com temperatura regulada para $25^{\circ} \mathrm{C}$. As avaliações do número de sementes germinadas foram realizadas diariamente para a obtenção do Índice de Velocidade de Germinação-IVG; aos 4 dias após a montagem do teste, computou-se o número de plântulas normais (Primeira Contagem de Germinação), de acordo com BRASIL (2009). Os resultados de germinação foram expressos em porcentagem de plântulas normais, com avaliação aos 10 dias.

O teste de envelhecimento acelerado foi conduzido de acordo com a metodologia recomendada pela Association of Official Seed Analysts - AOSA (1983). Oito repetições de 25 sementes por lote foram dispostas sobre uma tela de alumínio fixada a uma caixa de plástico (11 cm x $11 \mathrm{~cm} \times 3 \mathrm{~cm}$ ). Em cada caixa, foram colocados $40 \mathrm{~mL}$ de água, e em seguida mantidos em BOD a $42^{\circ} \mathrm{C}$ por 96 h. Após esse período, as sementes foram submetidas ao teste de germinação, conforme BRASIL (2009).

O teste de sanidade foi conduzido pelo método de incubação em papel-filtro sem congelamento (NEERGAARD, 1979) com 8 repetições de 25 sementes por lote. As sementes foram distribuídas em placa de Petri de $15 \mathrm{~cm}$ de diâmetro contendo três folhas de papel-filtro previamente esterilizadas e umedecidas em solução de 2,4-D a 10 ppm. As sementes foram incubadas a $20 \pm 2{ }^{\circ} \mathrm{C}$, em câmara com fotoperíodo de $12 \mathrm{~h}$, durante sete dias. Para a identificação de patógenos presentes nas sementes, foi utilizado lupa estereoscópica e microscópio óptico. A incidência foi avaliada em porcentagem de sementes com fungos.

No teste de tetrazólio, os aquênios foram imersos em água por $15 \mathrm{~h}$ a $25^{\circ} \mathrm{C}$, para a retirada do pericarpo. As sementes foram cortadas longitudinalmente entre os cotilédones até 0 centro da semente, conforme prescrição das Regras para Análise de Sementes da International Seed Testing Association - ISTA (2013). As sementes foram imersas em água destilada por 15 a 30 min, para a retirada da membrana aderida à semente. Posteriormente, as sementes foram colocadas na solução de tetrazólio na concentração de $0,075 \%$ e mantidas em estufa pelo período de uma hora, à temperatura de $35^{\circ} \mathrm{C}$. As sementes foram classificadas em viáveis e não viáveis, seguindo padrão proposto pela ISTA (2013).

O delineamento experimental utilizado foi o inteiramente casualizado. Os resultados foram comparados estatisticamente por meio de análise de variância, e as médias, comparadas pelo teste de Scott-Knott, a 5\% de probabilidade. Foram calculados os coeficientes de correlação entre os índices fornecidos pelo sistema computadorizado para análise de imagens de plântulas SVIS\& e os demais testes realizados.

\section{Resultados e discussão}

$\mathrm{Na}$ caracterização da qualidade dos lotes com sementes recém-colhidas (Tabela 1), os lotes 5 e 6 foram superiores na primeira contagem de germinação, sendo nestes lotes observada menor porcentagem de plântulas anormais infeccionadas. Para o índice de velocidade de germinação, não houve separação dos lotes em diferentes níveis de qualidade. Para o teste de tetrazólio, os lotes 1 e 5 foram considerados inferiores aos demais. A variação nos resultados pode ocorrer pela ação de fungos que interferem nos resultados dos testes que envolvem germinação em substrato de papel. O teste de tetrazólio apresenta limitações de utilização, justamente no caso de a espécie apresentar alto índice de patógenos associados. PINTO et al. (2009) também verificaram a interferência de patógenos na germinação de sementes de pinhão-manso e diferenças em relação aos resultados do teste de tetrazólio. Nos testes de envelhecimento acelerado, índice de velocidade de emergência e emergência final, não houve diferenciação dos lotes em níveis de qualidade. Para a primeira contagem de emergência, o lote 5 apresentou melhor qualidade fisiológica.

Após quatro meses de armazenamento, 
os lotes não diferiram quanto ao índice de velocidade de germinação, primeira contagem e tetrazólio (Tabela 2). Para germinação, os lotes 2 e 5 foram classificados como os de pior qualidade fisiológica; os lotes 2; 3 e 5 foram inferiores aos demais no teste de envelhecimento acelerado.

Tabela 1 - Valores médios de índice de velocidade de germinação - IVG, primeira contagem de germinação - $\mathrm{PC}(\%)$, germinação - $\mathrm{G}(\%)$, anormais infeccionadas - $\mathrm{Al}(\%)$, tetrazólio, envelhecimento acelerado - EA(\%), índice de velocidade de emergência - IVE, primeira contagem de emergência - PCE $(\%)$ e emergência final - EF(\%), de sementes de girassol recém-colhidas. Germination speed index (IVG), germination first count (PC - \%), germination (G - \%), infected abnormals (AI - \%), tetrazolium, accelerated aging (EA - \%), emergence speed index (IVE), emergence first count (PCE $\%)$, and final emergence (EF - \%) of recently harvested sunflower seed.

\begin{tabular}{ccccrrrrrr}
\hline Lote & $\mathrm{IVG}^{*}$ & $\mathrm{PC}$ & $\mathrm{G}$ & $\mathrm{Al}$ & \multicolumn{1}{c}{$\mathrm{TZ}$} & $\mathrm{EA}^{*}$ & $\mathrm{IVE}^{*}$ & $\mathrm{PCE}$ & $\mathrm{EF}^{*}$ \\
\hline 1 & 11,77 & $66 \mathrm{~b}$ & $80 \mathrm{~b}$ & $19 \mathrm{a}$ & $95 \mathrm{~b}$ & 71 & 8,67 & $90 \mathrm{~b}$ & 92 \\
2 & 11,54 & $65 \mathrm{~b}$ & $76 \mathrm{~b}$ & $18 \mathrm{a}$ & $100 \mathrm{a}$ & 75 & 8,52 & $87 \mathrm{~b}$ & 88 \\
3 & 11,50 & $67 \mathrm{~b}$ & $81 \mathrm{~b}$ & $15 \mathrm{a}$ & $100 \mathrm{a}$ & 73 & 8,69 & $90 \mathrm{~b}$ & 91 \\
4 & 11,27 & $57 \mathrm{c}$ & $76 \mathrm{~b}$ & $20 \mathrm{a}$ & $100 \mathrm{a}$ & 75 & 8,42 & $85 \mathrm{~b}$ & 88 \\
5 & 11,96 & $74 \mathrm{a}$ & $91 \mathrm{a}$ & $7 \mathrm{~b}$ & $94 \mathrm{~b}$ & 76 & 9,23 & $95 \mathrm{a}$ & 96 \\
6 & 11,79 & $75 \mathrm{a}$ & $88 \mathrm{a}$ & $9 \mathrm{~b}$ & $98 \mathrm{a}$ & 78 & 8,86 & $89 \mathrm{~b}$ & 93 \\
\hline $\mathrm{CV}(\%)$ & & 11,62 & 8,06 & 48,24 & 2,10 & & & 3,36 & \\
\hline
\end{tabular}

Médias seguidas da mesma letra não diferem entre si, pelo teste de Scott-Knott, a $5 \%$ de probabilidade. * Médias não diferem entre si, pelo teste $\mathrm{F}$, a $5 \%$ de probabilidade.

Tabela 2 - Valores médios de índice de velocidade de germinação - IVG, primeira contagem de germinação - $\mathrm{PC}(\%)$, germinação - $\mathrm{G}(\%)$, anormais infeccionadas - $\mathrm{Al}(\%)$ e tetrazólio - $\mathrm{TZ}(\%)$, envelhecimento acelerado - EA(\%), índice de velocidade de emergência - IVE, primeira contagem de emergência - PCE(\%) e emergência final - $E F(\%)$, de sementes de girassol após quatro meses de armazenamento. Germination speed index (IVG), germination first count (PC - \%), germination (G - \%), infected abnormals (AI - \%), tetrazolium, accelerated aging (EA - \%), emergence speed index (IVE), emergence first count (PCE - \%), and final emergence (EF - \%) of sunflower seed after a four month storage period.

\begin{tabular}{cccccccccc}
\hline Lote & $\mathrm{IVG}^{*}$ & $\mathrm{PC}$ & $\mathrm{G}$ & $\mathrm{Al}$ & $\mathrm{TZ}^{*}$ & $\mathrm{EA}$ & $\mathrm{IVE}^{*}$ & $\mathrm{PCE}^{*}$ & $\mathrm{EF}$ \\
\hline 1 & 11,64 & $55 \mathrm{a}$ & $66 \mathrm{a}$ & $30 \mathrm{a}$ & 98 & $59 \mathrm{a}$ & 9,95 & 82 & $83 \mathrm{a}$ \\
2 & 11,76 & $51 \mathrm{a}$ & $60 \mathrm{~b}$ & $35 \mathrm{a}$ & 96 & $50 \mathrm{~b}$ & 9,12 & 77 & $77 \mathrm{a}$ \\
3 & 11,60 & $53 \mathrm{a}$ & $68 \mathrm{a}$ & $21 \mathrm{~b}$ & 99 & $47 \mathrm{~b}$ & 10,41 & 83 & $84 \mathrm{a}$ \\
4 & 11,64 & $62 \mathrm{a}$ & $75 \mathrm{a}$ & $21 \mathrm{~b}$ & 97 & $67 \mathrm{a}$ & 9,79 & 87 & $88 \mathrm{a}$ \\
5 & 11,58 & $41 \mathrm{a}$ & $56 \mathrm{~b}$ & $38 \mathrm{a}$ & 99 & $55 \mathrm{~b}$ & 10,24 & 79 & $80 \mathrm{a}$ \\
6 & 12,05 & $60 \mathrm{a}$ & $65 \mathrm{a}$ & $32 \mathrm{a}$ & 97 & $61 \mathrm{a}$ & 9,34 & 85 & $86 \mathrm{a}$ \\
\hline $\mathrm{CV}(\%)$ & & 24,93 & 15,14 & 31,07 & & 17,57 & & & 5,65 \\
\hline
\end{tabular}

Médias seguidas da mesma letra não diferem entre si, pelo teste de Scott-Knott, a $5 \%$ de probabilidade. * Médias não diferem entre si, pelo teste $F$, a $5 \%$ de probabilidade.

As diferenças em termos de variação da qualidade dos lotes, antes e após o armazenamento, justificam-se principalmente pela incidência de Alternaria sp. nas sementes recém-colhidas (Tabela 3). Esse fungo, segundo HUMPHERSONJONES (1992), pode afetar negativamente a germinação por seus danos causados às plântulas. Observou-se pelos resultados do teste de sanidade que a incidência de Alternaria sp. foi reduzida com 0 armazenamento, resultados esperados, uma vez que fungos de campo tendem a diminuir seu potencial de infecção ao longo do armazenamento (TANAKA et al., 2001). Por outro lado, os fungos de armazenamento tendem a aumentar ao longo do tempo, o que pode ser confirmado pelo aumento do percentual de incidência de Aspergillus spp. (MEDINA et al., 2009). Para sementes recém-colhidas, não houve diferença significativa em relação à porcentagem de fungos Aspergillus spp. e Alternaria sp. nos diferentes lotes, exceto para o lote 6 , onde se observou incidência inferior de Alternaria sp, o que poderia explicar a alta germinação deste lote nas sementes recém-colhidas.

$\mathrm{Na}$ análise geral dos resultados, para as sementes armazenadas, observou-se qualidade fisiológica inferior dos lotes 2 e 5 , e superior do lote 4. Um dos aspectos que podem ter afetado a germinação das sementes do lote 5 , que para sementes recém-colhidas era considerado de 
qualidade superior em relação aos demais, foi a qualidade sanitária do lote, que apresentou a maior incidência de Aspergillus spp. e Alternaria sp.
(Tabela 3). Para os demais lotes, não foi observada diferença marcante na incidência de fungos.

Tabela 3 - Porcentagem (\%) dos fungos Aspergillus spp. e Alternaria sp. em seis lotes de sementes de girassol recém-colhidas e armazenadas por quatro meses. Percentage of Aspergillus spp. and Alternaria sp. fungi in sunflower seeds of six lots of recently harvested and after a four month period of storage.

\begin{tabular}{ccccc}
\hline \multirow{2}{*}{ Lote } & \multicolumn{2}{c}{ Aspergillus spp. } & \multicolumn{2}{c}{ Alternaria sp. } \\
\cline { 2 - 5 } & Recém-colhidas & Armazenadas & Recém-colhidas & Armazenadas \\
\hline 1 & 15 & 24 & 24 & 1 \\
2 & 21 & 27 & 30 & 6 \\
3 & 20 & 23 & 35 & 4 \\
4 & 18 & 26 & 36 & 8 \\
5 & 19 & 38 & 30 & 23 \\
6 & 18 & 17 & 20 & 11 \\
\hline
\end{tabular}

Os resultados do índice de vigor, de uniformidade de desenvolvimento, de crescimento e de comprimento de plântulas obtidos pelo SVIS® para as sementes recém-colhidas são apresentados na Tabela 4. De modo geral, a avaliação das plântulas com dois dias após a semeadura não possibilitou a separação de lotes em níveis de qualidade, exceção feita para o comprimento de plântulas que possibilitou a distinção dos lotes 3; 4 e 5 como os de qualidade superior, resultados esses não coincidentes com a classificação dos demais testes. Aos três dias de avaliação para o indicie de vigor (IV3), de crescimento (IC3) e comprimento de plântulas (CP3), os lotes 4; 5 e 6 foram menos vigorosos, resultados que não coincidem com as avaliações tradicionais efetuadas antes do armazenamento. A análise computadorizada SVISß não detectou diferenças significativas entre os índices de uniformidade calculados. Esses resultados permitem inferir que há necessidade de melhor adequação do programa usado para sementes de girassol.

Tabela 4 - Valores médios de índice de vigor - IV; de uniformidade de desenvolvimento - IU; de crescimento - IC e comprimento de plântulas (cm) - CP, para os diferentes tipos de avaliação: (2) avaliação aos 2 dias de germinação; (3) avaliação aos 3 dias de germinação, de seis lotes de sementes de girassol recém-colhidas. Vigor index (IV), development uniformity (IU), growth uniformity (IC), and seedling length (cm) (CP) from two evaluations [the first (2) at two days of germination, and the second (3) at three days of germination] of six recently harvested lots of sunflower seeds.

\begin{tabular}{ccccc}
\hline Lote & IV2 $^{*}$ & IU2 $^{*}$ & IC2 $^{*}$ & CP2 \\
\hline 1 & 564 & 831 & 449 & $1,29 \mathrm{~b}$ \\
2 & 591 & 843 & 483 & $1,40 \mathrm{~b}$ \\
3 & 633 & 865 & 534 & $1,64 \mathrm{a}$ \\
4 & 673 & 861 & 593 & $1,80 \mathrm{a}$ \\
5 & 624 & 853 & 527 & $1,39 \mathrm{~b}$ \\
6 & 583 & 877 & 457 & 13,59 \\
\hline CV(\%) & & & CP3 \\
\hline Lote & IV3 & IU3* & $5,35 \mathrm{~b}$ \\
2 & $776 \mathrm{~b}$ & 831 & $753 \mathrm{~b}$ & $5,04 \mathrm{~b}$ \\
3 & $743 \mathrm{~b}$ & 825 & $709 \mathrm{~b}$ & $6,43 \mathrm{a}$ \\
5 & $875 \mathrm{a}$ & 828 & $896 \mathrm{a}$ & $4,17 \mathrm{c}$ \\
6 & $608 \mathrm{c}$ & 831 & $513 \mathrm{c}$ & $4,61 \mathrm{c}$ \\
\hline CV(\%) & $651 \mathrm{c}$ & 848 & $568 \mathrm{c}$ & $4,49 \mathrm{c}$ \\
\hline
\end{tabular}

Médias seguidas da mesma letra não diferem entre si, pelo teste de Scott-Knott, a $5 \%$ de probabilidade. * Médias não diferem entre si, pelo teste $\mathrm{F}$, a $5 \%$ de probabilidade.

Após o armazenamento, não houve distinção dos lotes em níveis de qualidade para

os índices de vigor IV2 e IV3. O mesmo pode ser observado para os índices de uniformidade IU2 e 
IU3, comprimento de plântulas CP2 e CP3 e índices de crescimento IC2 e IC3 (Tabela 5). Para as sementes armazenadas, conforme observado para as sementes recém-colhidas, não foi possível a classificação dos lotes pelo SVIS® de maneira semelhante aos demais testes utilizados para a avaliação da qualidade de sementes.

Tabela 5 - Valores médios de índice de vigor - IV; de uniformidade de desenvolvimento - IU; de crescimento - IC e comprimento de plântulas $(\mathrm{cm})$ - CP, para os diferentes tipos de avaliação: (2) avaliação aos 2 dias de germinação; (3) avaliação aos 3 dias de germinação, de seis lotes de sementes de girassol após quatro meses de armazenamento. Vigor index (IV), development uniformity (IU), growth uniformity (IC), and seedling length $(\mathrm{cm})(C P)$ from two evaluations [the first (2) at two days of germination, and the second (3) at three days of germination] of six lots of sunflower seeds stored for a period of 4 months.

\begin{tabular}{cllll}
\hline Lote & IV2 $^{*}$ & IU2 $^{*}$ & IC2 $^{*}$ & CP2 $^{*}$ \\
\hline 1 & 739 & 862 & 687 & 2,08 \\
2 & 683 & 859 & 608 & 1,86 \\
3 & 681 & 836 & 616 & 1,87 \\
4 & 702 & 841 & 643 & 1,93 \\
5 & 707 & 868 & 638 & 1,94 \\
6 & 660 & 855 & 577 & 1,75 \\
\hline Lote & IV3 & IU3 & IC3 & CP3 \\
\hline 1 & 672 & 828 & 606 & 4,92 \\
2 & 726 & 845 & 676 & 5,55 \\
3 & 706 & 817 & 659 & 5,35 \\
4 & 679 & 840 & 610 & 5,02 \\
5 & 690 & 852 & 621 & 5,05 \\
6 & 659 & 829 & 587 & 4,80 \\
\hline
\end{tabular}

* Médias não diferem entre si, pelo teste $\mathrm{F}$, a $5 \%$ de probabilidade.

Os resultados obtidos com a utilização do software SVIS® não foram coerentes com os testes utilizados para a avaliação da qualidade inicial das sementes. Os índices calculados pelo SVIS $\AA^{8}$ não propiciaram classificações semeIhantes dos lotes; sendo que, de maneira geral, não houve correlação significativa com os testes (Tabelas 6 e 7). As sementes de girassol, por serem oleaginosas, são mais suscetíveis aos patógenos (JOKER \& JEPSEN, 2003). GOMES et al. (2006) verificaram que a presença de fungos em sementes de girassol estava relacionada aos baixos percentuais de vigor e germinação, e alto índice de plântulas anormais infeccionadas. Entretanto, como as avaliações no SVIS® ocorreram com plântulas de 2 e 3 dias de germinação, ainda não havia desenvolvimento do fungo no papel de germinação de contaminação das plântulas. Segundo PIVETA et al. (2010), os microrganismos podem causar anormalidades e lesões nas plântulas, bem como deterioração de sementes, principalmente em testes realizados em incubadoras ou germinadores, que dão condições ideais para o desenvolvimento e a disseminação de alguns fungos, causando apodrecimento das sementes e dificultando o diagnóstico correto da qualidade. A influência dos fungos nos resultados dos testes de avaliação da qualidade inicial das sementes, fato não observado na avaliação pelo SVIS®, pode ser um dos motivos da discordância dos resultados.

O Sistema de análise de imagens SVIS ${ }^{\circledR}$ foi considerado promissor para diversas espécies, como alface (PEÑALOZA et al., 2005; SAKO et al., 2001), melão (MARCOS FILHO et al., 2006), milho (HOFFMASTER et al., 2005; OTONI \& MCDONALD, 2005), algodão (OTONI et al., 2010) e soja (HOFFMASTER et al., 2003, 2005; MARCOS FILHO et al., 2009). TOHIDLOO \& KRUSE (2009), com sementes de colza (Brassica napus L.), verificaram que a avaliação do vigor pelo SVIS® não foi eficiente. O crescimento e as características morfológicas das plântulas precisam ser levados em consideração ao adaptar-se as diferentes espécies ao tipo de sistema de análise de imagem (TOHIDLOO \& KRUSE, 2009).

O sistema computadorizado de análise de plântulas SVIS $\circledast$ não possibilitou a diferenciação da qualidade entre os lotes de sementes de girassol, necessitando, assim, de estudos adicionais com refinamento das técnicas para verificação de sua utilização para sementes desta espécie. 
Tabela 6 - Coeficientes de correlação simples de Pearson $(r)$ estimados entre os testes de Índice de Velocidade de Germinação- IVG, Primeira contagem de germinação - PC, Germinação - G, Anormais infeccionadas - AI, Envelhecimento acelerado - EA, Índice de velocidade de emergência - IVE, Primeira contagem de emergência - PCE, Emergência final - EF e Tetrazólio, e os índices calculados pelo SVIS, para seis lotes de sementes de girassol recém-colhidas. Estimated simple Pearson correlation coefficients between results of the speed of germination (IVG), first count of germination (PC), germination (G), infected abnormals (AI), accelerated aging (EA), speed of emergence index (IVE), emergence first count (PCE), final emergence (EF), tetrazolium, and the indexes calculated by the SVIS for six recently harvested lots of sunflower seeds.

\begin{tabular}{|c|c|c|c|c|c|c|c|c|c|}
\hline & IVG & $\mathrm{PC}$ & $\mathrm{G}$ & $\mathrm{Al}$ & EA & IVE & PCE & $\mathrm{EF}$ & TZ \\
\hline IV2 & $-0,376$ & $-0,216$ & $-0,061$ & $-0,065$ & $0,497^{\circ}$ & $-0,211$ & $-0,310$ & $-0,193$ & 0,327 \\
\hline IU2 & $-0,102$ & $-0,016$ & 0,260 & $-0,274$ & 0,179 & 0,075 & 0,149 & 0,222 & 0,169 \\
\hline CP2 & $-0,380$ & $-0,184$ & $-0,070$ & $-0,067$ & $0,481^{\circ}$ & $-0,199$ & $-0,324$ & $-0,181$ & 0,305 \\
\hline IC2 & $-0,378$ & $-0,223$ & $-0,108$ & $-0,026$ & $0,498^{\circ}$ & $-0,237$ & $-0,352$ & $-0,242$ & 0,313 \\
\hline IV3 & 0,091 & 0,020 & $-0,045$ & 0,077 & $-0,311$ & 0,126 & 0,228 & 0,082 & 0,164 \\
\hline IU3 & 0,101 & $-0,071$ & 0,143 & $-0,072$ & 0,106 & 0,330 & 0,299 & 0,158 & $-0,174$ \\
\hline CP3 & 0,080 & 0,025 & $-0,065$ & 0,091 & $-0,327$ & 0,103 & 0,206 & 0,076 & 0,195 \\
\hline IC3 & 0,045 & $-0,027$ & $-0,177$ & 0,188 & $-0,377$ & 0,031 & 0,125 & $-0,030$ & 0,196 \\
\hline
\end{tabular}

Tabela 7 - Coeficientes de correlação simples de Pearson ( $r$ ) estimados entre os testes de Índice de Velocidade de Germinação- IVG, Primeira contagem de germinação - PC, Germinação - G, Anormais infeccionadas - AI, Envelhecimento acelerado - EA, Îndice de velocidade de emergência - IVE, Primeira contagem de emergência- PCE, Emergência final - EF e Tetrazólio, e os índices calculados pelo SVIS, para seis lotes de sementes de girassol, após quatro meses de armazenamento. Estimated simple Pearson correlation coefficients between results of the speed of germination (IVG), first count of germination (PC), germination (G), infected abnormals $(A l)$, accelerated aging $(E A)$, speed of emergence index (IVE), emergence first count (PCE), final emergence (EF), tetrazolium, and the indexes calculated by the SVIS for six lots of sunflower seeds stored for four months.

\begin{tabular}{|c|c|c|c|c|c|c|c|c|c|}
\hline & IVG & PC & $G$ & $\mathrm{Al}$ & EA & IVE & PCE & EF & TZ \\
\hline IV2 & $-0,300$ & 0,003 & 0,004 & $-0,103$ & $-0,258$ & 0,153 & 0,172 & 0,166 & 0,016 \\
\hline IU2 & $-0,317$ & $-0,122$ & $-0,372$ & 0,258 & 0,006 & $-0,017$ & $-0,011$ & $-0,030$ & $-0,191$ \\
\hline CP2 & $-0,277$ & 0,029 & 0,058 & $-0,150$ & $-0,265$ & 0,170 & 0,193 & 0,190 & 0,060 \\
\hline IC2 & $-0,257$ & 0,034 & 0,097 & $-0,180$ & $-0,290$ & 0,176 & 0,196 & 0,194 & 0,064 \\
\hline IV3 & 0,251 & $-0,007$ & 0,097 & $-0,031$ & 0,126 & 0,026 & 0,135 & 0,104 & $-0,360$ \\
\hline IU3 & 0,217 & 0,108 & 0,189 & $-0,151$ & 0,319 & $-0,237$ & $-0,168$ & $-0,193$ & $-0,083$ \\
\hline CP3 & 0,224 & 0,021 & 0,121 & $-0,058$ & 0,096 & 0,086 & 0,195 & 0,169 & $-0,394$ \\
\hline IC3 & 0,209 & 0,006 & 0,094 & $-0,035$ & 0,080 & 0,078 & 0,182 & 0,153 & $-0,375$ \\
\hline
\end{tabular}

${ }^{*}=\mathrm{r}$ significativo a $5 \%$ de probabilidade

\section{Conclusões}

A análise computadorizada de imagens de plântulas de girassol, provenientes do teste de germinação com o uso do software SVIS $\AA$, desenvolvido para soja, não apresenta resultados compatíveis com os demais testes utilizados na avaliação da qualidade de sementes.

\section{Referências}

BRASIL. Ministério da Agricultura Pecuária e Abastecimento. Regras para análise de sementes. Brasília: SNDA/DNDV/CLAV, 2009. 220p.

FAGUNDES, M. H. Sementes de girassol: alguns comentários. Brasília, 2009. Disponível em: <http://www.conab.gov.br/download/cas/ especiais/Semente-de-Girassol.pdf $>$. Acesso em: 17 jan.2014.
GOMES, D. P.; BRINGEL, J. M. M.; MORAES, M. F. H.; GOMES, J. J. A.; LEITE, R. M.V. B. C. Qualidade fisiológica e sanitária de sementes de girassol cultivadas em Timon, MA. Summa Phytopathologica, Botucatu, v.32, n.3, p.291293, jul./set. 2006.

HOFFMASTER, A.L.; FUJIMURA, K.; MCDONALD, M.B.; BENNETT, M.A. An automated system for vigor testing three-day-old soybean seedlings. Seed Science and Technology, Zurich, v.31, n.3, p.701-713, Oct. 2003.

HOFFMASTER, A. F.; XU, L.; FUJIMURA, K.; MCDONALD, M.B.; BENNETT, M.A.; EVANS, A.F. The Ohio State University seed vigor imaging system (SVIS) for soybean and corn seedlings. Journal of Seed Technology, Lincoln, v.27, n.1, p.7-24, Jan. 2005. 
HUMPHERSON-JONES, F. M. Epidemiology and control of dark leaf spot of brassicas. In: CHELKOWSKI, J.; VISCONTI, A. (Ed.). Alternaria: biology, plant diseases and metabolites. Amsterdam: Elsevier, 1992. p.267-288.

INTERNATIONAL SEED TESTING ASSOCIATION. International rules for seed testing. Zurich, 2013. Disponível em: <http://www.seedtest.org/en/international-rulesfor-seed-testing-_content-1-1083-238.html>. Acesso em: 12 jān. 2014.

JOKER, D.; JEPSEN, J. Jatropha curcas L. seed leaflet. Humleback, Denmark, v.83, n.2, p.1-2, 2003.

MAGUIRE, J. D. Speeds of germination aid selection and evaluation for seedling emergence and vigor. Crop Science, Madison, v.2, p.176177, 1962.

MARCOS FILHO, J. ; BENNETT, M. A ; EVANS, A. S. ; GRASSBAUGH, E. M. Assessment of melon seed vigour by an automated computer imaging system compared to traditional procedures. Seed Science and Technology, Zurich, v.34, n.2, p.507-519, Mar. 2006.

MARCOS FILHO, J.; KIKUTI, A. L. P.; LIMA, L. $B$. Métodos para avaliação do vigor de sementes de soja, incluindo a análise computadorizada de imagens. Revista Brasileira de Sementes, Viçosa, MG, v.31, n.1, p.102-112, jan./fev. 2009.

MEDINA, P. F.; TAKANAKA, M. A. S.; PARISI, J. J. D. Sobrevivência de fungos associados ao potencial fisiológico de sementes de triticale ( $X$. Triticosecale Wittmack) durante o armazenamento. Revista Brasileira de Sementes, Viçosa, MG, v.31, n.4, p.17-26, jul./ago. 2009.

NEERGAARD, P. Seed pathology. London: The MacMillan, 1979. v.1, 839p.

OTONI, R. R.; MCDONALD, M. B. Moisture and temperature effects on maize and soybean seedlings using the seed vigor imaging system. Journal of Seed Technology, Lincoln, v.27, n.2, p.243-247, 2005.
OTONI, R. R.; MCDONALD, M. B.; TAY, D. The use of X-Rays for seed vigor classification of cotton seeds. Journal of Seed Technology, Lincoln, 2010. In press.

PEÑALOZA, P.; RAMIRES-ROSALES, G.; MCDONALD, M. B.; BENNET, M. A. Lettuce (Lactuca sativa L.) seed quality evaluation using seed physical attributes, saturated salt accelerated aging and the seed vigour imagung system. Eletronic Journal of Biotechnology, Valparaíso, v.8, n.3, p.1-9, 2005.

PINTO, T.L.F.; MARCOS FILHO, J.; FORTI, V.A.; CARVALHO, C.; GOMES JUNIOR, F. G. Avaliação da viabilidade de sementes de pinhão manso pelos testes de tetrazólio e de raios $X$. Revista Brasileira de Sementes, Viçosa, MG, v.31, n.2, p.195-2001, mar./abr. 2009.

PIVETA, G.; MENEZES, V. O.; PEDROSO, D. C.; MUNIZ, M. F. B.; BLUME, E. WIELEWICKI, A. P. Superação de dormência na qualidade de sementes e mudas: influência na produção de Senna multijuga (L. C. Rich.) Irwin \& Barneby. Acta Amazônica, Manaus, v.40, n.2, p.281-288, 2010.

SAKO, Y.; MCDONALD, M.B.; FUJIMURA, K.; EVANS, A.F.; BENNETT, M.A. A system for automated seed vigour assessment. Seed Science and Technology, Zurich, v.29, n.3, p.625636, Sept. 2001.

TANAKA, M. A. S.; MAEDA, J. A.; PLAZAS, I. A. H. Z. Microflora fúngica de sementes de milho em ambientes de armazenamento. Scientia Agricola, Piracicaba, v.58, n.3, p.501-508, jul./set. 2001.

TOHIDLOO, G.; KRUSE, M. Development of an image analysis aided seedling growth test for winter oilseed rape and verifi cation as a vigour test. Seed Science and Technology, Zurich, v.37, n.1, p.98-109, Apr. 2009.

VIEIRA, M. G. G. C.; CARVALHO, M. L. M.; MACHADO, J. C. Controle de qualidade de sementes. Lavras: UFLA/FAEPE, 1999. 113p. 